\title{
AOR
}

Selected Papers of \#AolR2020: The $21^{\text {st }}$ Annual Conference of the Association of Internet Researchers Virtual Event / 27-31 October 2020

\section{SKIPPING DISCOVERY? MUSIC DISCOVERY AND PERSONAL MUSIC COLLECTIONS IN THE STREAMING ERA}

Jack Ellis

University of Tasmania

\section{Introduction}

Online music streaming currently constitutes the preferred method of consumption among music listeners worldwide (International Federation of the Phonographic Industry [IFPI], 2019). These streaming services afford users unparalleled access to vast music databases, providing an abundance of music choice and possibilities for exploring and discovering new music. Within these platform environments, combinations of algorithmic recommendation systems and playlist curation are made salient as tools to help users navigate the conditions of abundant choice and retain listeners through the personalised curation of song suggestions (Seaver, 2018; Fry, 2018; Lüders, 2019). Despite the promise of music streaming services for the discovery of new music, curated and personalised playlists have been found to trigger only short-term exposure and may even be detrimental to long-term experiences and enduring engagement between listeners and music which is new to them (Kjus, 2016; Morgan, 2019). These perspectives draw attention to the intersection of user listening behaviours and algorithmic recommendation systems in shaping experiences of music discovery.

Investigating this relationship in the case of Spotify, Lüders (2019) describes users as engaging in a form of 'non-linear finding', as combinations of personalised algorithmic music recommendations and curated playlists serve to 'push' users to 'always explore further' (p.11). These features provide ways for service users to expand their music tastes and indulge their curiosities through exposure to new sounds. However, these features were simultaneously recognised as the source of ephemeral music experiences, characterised by impatience, a loss of focus and tendencies to skip quickly through songs in order to listen to the next suggestion. It is here that Lüders (2019) identifies user practices of saving and collecting music by creating their own playlists as a strategy to mitigate the ephemeral experience of exploring music on Spotify. These practices are indicative of a 'will to archive' among Spotify users as a way to exercise a sense of ownership over music and create connections between music and their own individualised histories (Lüders, 2019). Foregrounding the position of personal music collections in establishing enduring relationships with music on Spotify, Lüders' work 
may be complimented by scrutinising the 'phenomenological moment' of discovery itself (Nowak, 2016 p.140).

As such, in this paper I will locate the use of personal music collections by Spotify users to cultivate instances of music discovery which extend beyond initial encounters with music recommendation systems. This addition allows for the affective processes in which music becomes meaningful to be captured, as well as the evolving trajectories of these meanings over time. In doing so, I will engage with conference themes concerning platforms and algorithms and their increasing presence in shaping the distribution, consumption and discovery of culture in the everyday lives of individuals.

\section{Theoretical Framework}

My focus on 'phenomenological moments' of discovery follows Nowak's (2016) reframing of music discovery to include affective responses to music content which mediate the interpretation and definition of music by individuals. Nowak's conceptualisation explains how 'the initial interaction with content matters less than the interaction that leaves an affective mark on individuals — the actual discovery' (2016, p.143). In this way, an individual's social surroundings or the availability technologies for discovering music represent the conditions from which music discoveries may emerge, but these moments are modulated by affect, or the 'bodily responses to music content that arouse individuals' sensibilities and enable them to categorize content they discover' (Nowak, 2016 p.142). From this perspective, I examine how Spotify users experience different types of music discovery through their personal music collections, including growing music discoveries which emerge over time.

\section{Method}

The data for this paper was collected as part of a larger project focused on music collections. A series of semi-structured qualitative interviews were conducted with a research sample of 20 Generation Y music fans who use music streaming platforms, reported an active interest in popular music and ownership of at least one other music media format. Participants were recruited using an online Facebook advertisement. Interviews were recorded using a digital audio recorder before being transcribed and thematically coded (Saldaña, 2013).

\section{Findings}

A subset of my participants identified a change in their listening habits when using Spotify, reporting an excessive increase in the skipping of songs and impatience with song recommendations. In this context, the cultivation of a music collection became a strategy to mitigate feelings of being pushed to explore new music by platform interfaces and to slow down the pace of their music consumption. Such collections often took the form of user-created playlists explicitly intended for saving music recommendations from friends and others or suggested by service algorithms. Users consciously returned to these personal music archives with an intention to listen to music content more closely or in its entirety, constituting a listening context and mindset sympathetic towards affective response and different types of music discovery. These 
collections were described as the site of music which grew to be discovered over time through repeated listening and gradual affective definition by the listener. This process of emergent discovery made salient the influence of the listener's social surroundings, friends and online communities as the impetus for choosing to give particular music content time to grow towards being discovered.

\section{Conclusion}

This paper investigates the notion of music discovery at the current height of the streaming era of music consumption, where systems of service personalisation, recommendation and access to vast cloud-based music databases shape the practices of a significant proportion of music consumers. Music discoveries were found to represent a nexus of culture, algorithms, listener practices and social engagements. Adopting a focus on user practices of personal archiving and music management within the architecture of music streaming services, I locate personal music collections as the site of an enduring 'will to archive', in order to remember music which is important and facilitate affective musical discoveries (Lüders, 2019). This focus yielded longitudinal perspectives on music discovery which reflected long-term experiences and sustained engagement between users and music which is new to them. These findings raise implications for the use of algorithmic recommendation systems in the delivery and consumption of music and other cultural forms, drawing attention to user practices and strategies as platforms and algorithms become increasingly relevant conduits of cultural diffusion and circulation.

\section{References}

Fry, A. (2018). An Echoic Chamber: Algorithmic Curation and Personalized Listening. In: E. Mazierska, L. Gillon and T. Rigg, ed., Popular Music in the Post-Digital Age Politics, Economy, Culture and Technology, 1st ed. New York: Bloomsbury Academic, pp.269-290.

IFPI. (2019). IFPI Global Music Report 2019. [online] Available at: https://www.ifpi.org/news/IFPI-GLOBAL-MUSIC-REPORT-2019 [Accessed 28 Feb. 2020].

Kjus, Y. (2016). Musical exploration via streaming services: The Norwegian experience. Popular Communication, 14(3), pp.127-136.

Lüders, M. (2019). Pushing music: People's continued will to archive versus Spotify's will to make them explore. European Journal of Cultural Studies, pp.1-18.

Morgan, B. (2019). Revenue, access, and engagement via the in-house curated Spotify playlist in Australia. Popular Communication, 18(1), pp.32-47.

Nowak, R. (2016). When is a discovery? The affective dimensions of discovery in music consumption. Popular Communication, 14(3), pp.137-145. 
Saldaña, J. (2009). The Coding Manual for Qualitative Researchers. 1st ed. London: SAGE.

Seaver, N. (2018). Captivating algorithms: Recommender systems as traps. Journal of Material Culture, 24(4), pp.421-436. 